\title{
Synthesis of Dimethyl Sulfomycinamate
}

\author{
Mark C. Bagley, ${ }^{* a}$ James W. Dale, ${ }^{a}$ Xin Xiong ${ }^{a}$ and Justin Bower ${ }^{b}$ \\ ${ }^{a}$ Department of Chemistry, Cardiff University, PO Box 912, Cardiff, CF10 3TB, UK. \\ ${ }^{b}$ Vernalis, Granta Park, Abington, Cambridge, CB1 6GB, UK. \\ Email AddressBagleymc@cf.ac.uk
}

\section{Ethyl 4-hydroxy-2-(2-propenyl)-2-oxazoline-4-carboxylate (6)}

Ethyl bromopyruvate $(0.9 \mathrm{ml}, 7.17 \mathrm{mmol})$ was added to a solution of methacrylamide (5) $(0.5 \mathrm{~g}, 6.02$ $\mathrm{mmol})$ and $\mathrm{NaHCO}_{3}{ }^{1}(2.5 \mathrm{~g}, 29.76 \mathrm{mmol})$ in dry tetrahydrofuran $(60 \mathrm{ml})$. The mixture was heated at reflux for $18 \mathrm{~h}$, filtered through Celite ${ }^{\circledR}$ and evaporated in vacuo. Purification by recrystallization (light petroleum-diethyl ether) gave the title compound as a colourless solid $(0.96 \mathrm{~g}, 80 \%), \mathrm{mp} 80-81{ }^{\circ} \mathrm{C}$ (ethyl acetate) (Found: $\mathrm{C}, 54.0 ; \mathrm{H}, 6.4 ; \mathrm{N}$, 6.7. Calc. for $\mathrm{C}_{9} \mathrm{H}_{13} \mathrm{NO}_{4}$ : C, 54.3; H, 6.6; N, 7.0\%) (Found: $\mathrm{MH}^{+}, 200.0916 . \mathrm{C}_{9} \mathrm{H}_{13} \mathrm{NO}_{4}$ requires $\left.M H, 200.0917\right) ; v_{\max }($ nujol $) / \mathrm{cm}^{-1} 1749,1654,1602,1459,1376$, 1224, 1154, 1083, 1016 and 954; $\delta_{\mathrm{H}}\left(400 \mathrm{MHz} ; \mathrm{d}_{4}\right.$-methanol) $5.89(1 \mathrm{H}, \mathrm{m}, \mathrm{CHH}), 5.52(1 \mathrm{H}, \mathrm{m}, \mathrm{CH})$, $4.59(1 \mathrm{H}, \mathrm{d}, J 10.0, \mathrm{OCHH}), 4.15\left(2 \mathrm{H}, \mathrm{q}, J\right.$ 7.1, $\left.\mathrm{CH}_{2} \mathrm{Me}\right), 4.12(1 \mathrm{H}, \mathrm{d}, J 10.0, \mathrm{OCH} H), 1.86(3 \mathrm{H}, \mathrm{m}$, Me), $1.21\left(3 \mathrm{H}, \mathrm{t}, J\right.$ 7.1, $\left.\mathrm{CH}_{2} \mathrm{Me}\right)$; $\delta_{\mathrm{C}}\left(100 \mathrm{MHz}\right.$; $\mathrm{d}_{4}$-methanol) $170.6(\mathrm{C}), 168.6(\mathrm{C}), 132.2(\mathrm{C}), 123,8$ $\left(\mathrm{CH}_{2}\right), 97.1(\mathrm{C}), 76.1\left(\mathrm{CH}_{2}\right), 62.0\left(\mathrm{CH}_{2}\right), 17.9(\mathrm{Me})$ and $13.0(\mathrm{Me}) ; \mathrm{m} / z(\mathrm{APcI}) 200\left(\mathrm{MH}^{+}, 100 \%\right)$ and $182(32)$.

\section{Ethyl 2-(2-propenyl)oxazole-4-carboxylate (7)}

A solution of 2,6-lutidine $(8.12 \mathrm{ml}, 77.12 \mathrm{mmol})$ and trifluoroacetic anhydride $(4.73 \mathrm{ml}, 33.49 \mathrm{mmol})$ in dry tetrahydrofuran $(10 \mathrm{ml})$ was added to a solution of ethyl 4-hydroxy-2-(2-propenyl)-2-oxazoline4-carboxylate (6) $(5.55 \mathrm{~g}, 27.86 \mathrm{mmol})$ at $0{ }^{\circ} \mathrm{C}$. After stirring for 30 minutes, water $(50 \mathrm{ml})$ was added and the mixture was concentrated in vacuo. Purification by flash chromatography on silica, gradient eluting with light petroleum to light petroleum-ethyl acetate $(3: 1)$, gave the title compound as a colourless oil (5.06 g, 94\%) (Found: $\mathrm{MH}^{+}$, 182.0810. $\mathrm{C}_{9} \mathrm{H}_{11} \mathrm{NO}_{3}$ requires $M H$, 182.0817); $v_{\max }($ film $) / \mathrm{cm}^{-1} 3155,2984,1744,1575,1543,1448,1391,1315,1254,1176,115,982,916$ and 763; 
$\delta_{\mathrm{H}}\left(400 \mathrm{MHz} ; \mathrm{CDCl}_{3}\right) 8.12(1 \mathrm{H}, \mathrm{s}, 5-\mathrm{H}), 6.15(1 \mathrm{H}, \mathrm{d}, J$ 1.4, CHH), $5.39(1 \mathrm{H}, \mathrm{dd}, J$ 1.4, 0.9, CHH), 4.32 (2H, q, J 7.1, $\left.\mathrm{CH}_{2} \mathrm{Me}\right), 2.12(3 \mathrm{H}, \mathrm{s}, \mathrm{Me}), 1.30\left(3 \mathrm{H}, \mathrm{t}, J\right.$ 7.1, $\left.\mathrm{CH}_{2} \mathrm{Me}\right) ; \delta_{\mathrm{C}}\left(100 \mathrm{MHz} ; \mathrm{CDCl}_{3}\right) 163.1(\mathrm{C})$, $161.3(\mathrm{C}), 143.5(\mathrm{CH}), 134.2(\mathrm{C}), 131.1(\mathrm{C}), 119.9\left(\mathrm{CH}_{2}\right), 61.2\left(\mathrm{CH}_{2}\right), 19.0(\mathrm{Me})$ and $14.3(\mathrm{Me}) ; \mathrm{m} / z$ (EI) $181\left(\mathrm{M}^{+}, 100 \%\right)$.

\section{2-(2-Propenyl)oxazole-4-carboxylic acid (8)}

Lithium hydroxide monohydrate $(6.49 \mathrm{~g}, 0.155 \mathrm{M})$ was added to a solution of ethyl 2-(2propenyl)oxazole-4-carboxylate (7) (4.76 g, $26.44 \mathrm{mmol})$ in $\mathrm{MeOH}$-water (1:1) (100 ml). The mixture was stirred at room temperature for 2 hours, concentrated in vacuo and partitioned between water (100 $\mathrm{ml})$ and chloroform $(50 \mathrm{ml})$. The aqueous layer was further extracted with chloroform $(2 \times 50 \mathrm{ml})$, acidified to $\mathrm{pH}$ 2-3 with dilute hydrochloric acid $(3 \mathrm{~N})$ and extracted with chloroform (3 x $50 \mathrm{ml})$. The combined organic extracts were washed with brine $(25 \mathrm{ml})$, dried $\left(\mathrm{MgSO}_{4}\right)$ and evaporated in vacuo to give the title compound as a colourless solid (3.14 g, 78\%), mp 121.5-122 ${ }^{\circ} \mathrm{C}$ (ethyl acetate) (Found: $\mathrm{C}$, 54.6; H, 4.6; N, 8.9. Calc. for $\mathrm{C}_{9} \mathrm{H}_{13} \mathrm{NO}_{4}: \mathrm{C}, 54.9 ; \mathrm{H}, 4.6 ; \mathrm{N}, 9.2 \%$ ) (Found: $\mathrm{MH}^{+}$, 154.0498. $\mathrm{C}_{7} \mathrm{H}_{7} \mathrm{NO}_{3}$ requires $M H, 154.0499) ; v_{\max }\left(\right.$ nujol)/cm ${ }^{-1} 3136,1691,1562,1462,1377,1260,1186,1124,984,910$, 853, 766 and 665; $\delta_{\mathrm{H}}\left(400 \mathrm{MHz} ; \mathrm{CDCl}_{3}\right) 10.26\left(1 \mathrm{H}, \mathrm{bs}, \mathrm{CO}_{2} \mathrm{H}\right), 8.23(1 \mathrm{H}, \mathrm{s}, 5-\mathrm{H}), 6.00(1 \mathrm{H}, \mathrm{s}, \mathrm{CHH})$, $5.44(1 \mathrm{H}, \mathrm{s}, \mathrm{CH} H), 2.14(3 \mathrm{H}, \mathrm{s}, \mathrm{Me}) ; \delta_{\mathrm{C}}\left(100 \mathrm{MHz} ; \mathrm{CDCl}_{3}\right) 166.1(\mathrm{C}), 163.5(\mathrm{C}), 145.0(\mathrm{CH}), 133.4(\mathrm{C})$, $130.9(\mathrm{C}), 120.6\left(\mathrm{CH}_{2}\right)$ and $19.0(\mathrm{Me}) ; \mathrm{m} / z(\mathrm{APcI}) 154\left(\mathrm{MH}^{+}, 100 \%\right)$ and $136(80)$.

\section{$N$-Methoxy- $N$-methyl-2-(2-propenyl)oxazole-4-carboxamide (9)}

Ethyl chloroformate $(0.57 \mathrm{ml}, 6.01 \mathrm{mmol})$ was added dropwise to a solution of 2-(2-propenyl)oxazole4-carboxylic acid (8) (0.97 g, $6.96 \mathrm{mmol})$ and triethylamine $(0.97 \mathrm{ml}, 6.96 \mathrm{mmol})$ in dry tetrahydrofuran $(30 \mathrm{ml})$ at $0{ }^{\circ} \mathrm{C}$. After stirring for 30 minutes, $N, O$-dimethylhydroxlamine hydrochloride $(0.68 \mathrm{~g}, 6.97 \mathrm{mmol})$ was added and the mixture was stirred for 18 hours, concentrated in vасио and partitioned between water $(100 \mathrm{ml})$ and chloroform $(50 \mathrm{ml})$. The aqueous layer was further extracted with chloroform $(2 \times 50 \mathrm{ml})$ and the combined organic extracts were washed with brine (30 $\mathrm{ml})$, dried $\left(\mathrm{Na}_{2} \mathrm{SO}_{4}\right)$ and evaporated in vacuo. Purification by flash chromatography on silica, eluting with light petroleum-ethyl acetate $(1: 3)$, gave the title compound as a colourless oil $(0.81 \mathrm{~g}, 81 \%)^{2}$ (Found: $\mathrm{MH}^{+}, 197.0924 . \mathrm{C}_{9} \mathrm{H}_{12} \mathrm{~N}_{2} \mathrm{O}_{3}$, requires $\left.M H, 197.0926\right)$; $v_{\max }\left(\right.$ film)/cm ${ }^{-1} 3166,2976,2939,1653$, $1551,1422,1386,1353,1118,1082,998,965,869$ and 747; $\delta_{\mathrm{H}}\left(400 \mathrm{MHz} ; \mathrm{CDCl}_{3}\right) 8.07(1 \mathrm{H}, \mathrm{s}, 5-\mathrm{H})$,

\footnotetext{
${ }^{1} \mathrm{NaHCO}_{3}$ was ground and dried in a conventional oven at $115^{\circ} \mathrm{C}$ for two days before use

${ }^{2}$ Yield based on recovered starting material (20\%)
} 
5.95 (1H, m, CHH), 5.38 (1H, m, CHH), 3.70 (3H, s, OMe), 3.33 (3H, s, NMe), 2.14 (3H, s, 2'-Me); (100 MHz; $\left.\mathrm{CDCl}_{3}\right)$ 162.2 (C), $161.3(\mathrm{C}), 142.3(\mathrm{CH}), 134.3(\mathrm{C}), 131.3(\mathrm{C}), 119.4\left(\mathrm{CH}_{2}\right) 61.4(\mathrm{Me})$, $33.3(\mathrm{Me})$ and $19.1(\mathrm{Me}) ; \mathrm{m} / z$ (APcI) $197\left(\mathrm{MH}^{+}, 100 \%\right), 167(30)$.

\section{2-Methyl-4-(hydroxymethyl)thiazole}

Ethyl 2-methylthiazole-4-carboxylate ${ }^{3}(20.0 \mathrm{~g}, 0.117 \mathrm{~mol})$ was added portion wise to a solution of lithium aluminium hydride (1.0 $\mathrm{M}$ in tetrahydrofuran; $100 \mathrm{ml}, 0.1 \mathrm{~mol})$ in dry tetrahydrofuran $(350 \mathrm{ml})$. The mixture was stirred for 1.5 hours, quenched sequentially with water and dilute aqueous sodium hydroxide solution $(2 \mathrm{~N} ; 250 \mathrm{ml}$ ) and stirred vigorously for one hour. The organic layer was decanted and the residue was extracted with tetrahydrofuran $(3 \times 250 \mathrm{ml})$, stirring vigorously for 15 minutes each time. The combined organic extracts were dried $\left(\mathrm{MgSO}_{4}\right)$ and evaporated in vacuo to give 2methyl-4-(hydroxymethyl)thiazole as a red oil (9.06 g, 60\%) (Found: $\mathrm{MH}^{+}, 130.0328$. $\mathrm{C}_{5} \mathrm{H}_{7} \mathrm{NOS}$ requires $M H, 130.0326) ; v_{\max }\left(\right.$ film) $/ \mathrm{cm}^{-1} 3281,2924,2855,1532,1478,1438,1171,1130,857$ and 753 ; $\delta_{\mathrm{H}}\left(400 \mathrm{MHz} ; \mathrm{CDCl}_{3}\right) 6.94(1 \mathrm{H}, \mathrm{s}, 5-\mathrm{H}), 5.03(1 \mathrm{H}, \mathrm{bs}, \mathrm{OH}), 4.62\left(2 \mathrm{H}, \mathrm{s}, \mathrm{CH}_{2}\right), 2,59(3 \mathrm{H}, \mathrm{s}, \mathrm{Me}) ; \delta_{\mathrm{C}}(100$ $\left.\mathrm{MHz} ; \mathrm{CDCl}_{3}\right)$ 167.0 (C), $156.2(\mathrm{C}), 114.4(\mathrm{CH}), 60.0\left(\mathrm{CH}_{2}\right)$ and $18.9(\mathrm{Me}) ; \mathrm{m} / z(\mathrm{APcI}) 130\left(\mathrm{MH}^{+}, 42 \%\right)$ and $112(100)$.

\section{2-Methyl-4-[2-(trimethylsilyl)ethoxymethoxymethyl]thiazole (10)}

$N, N$-Diisopropylethylamine $(35 \mathrm{ml}, 0.20 \mathrm{~mol})$ was added dropwise over 1.5 hours to a solution of 2methyl-4-(hydroxymethyl)thiazole $(8.61 \mathrm{~g}, 66.7 \mathrm{mmol})$ and 2-(trimethylsilyl)ethoxymethyl chloride (SEM-Cl) $(23.6 \mathrm{ml}, 0.133 \mathrm{~mol})$ in dry dichloromethane $(500 \mathrm{ml})$. The mixture was stirred for a further hour, concentrated to $200 \mathrm{ml}$ in vacuo, washed sequentially with dilute hydrochloric acid (3 N; 2 x 200 $\mathrm{ml})$ and brine $(50 \mathrm{ml})$, dried $\left(\mathrm{MgSO}_{4}\right)$ and evaporated in vacuo. Purification by distillation gave the title compound as a clear oil (14.35 g, 83\%), bp 160-164 ${ }^{\circ} \mathrm{C}$ (2 Torr) (Found: $\mathrm{MH}^{+}, 260.1135$. $\mathrm{C}_{11} \mathrm{H}_{21} \mathrm{NO}_{2} \mathrm{SSi}$, requires $\left.M H, 260.1141\right) ; v_{\max }\left(\right.$ film) $/ \mathrm{cm}^{-1}$ 2952, 1885, 1248, 1184, 1107, 1061, 937, 860, 836 and 734; $\delta_{\mathrm{H}}\left(400 \mathrm{MHz} ; \mathrm{CDCl}_{3}\right) 7.04(1 \mathrm{H}, \mathrm{s}, 5-\mathrm{H}), 4.78\left(2 \mathrm{H}, \mathrm{s}, \mathrm{OCH}_{2} \mathrm{O}\right), 4.65\left(2 \mathrm{H}, \mathrm{s}, 4-\mathrm{CH}_{2}\right), 3.67$ $\left(2 \mathrm{H}, \mathrm{m}, \mathrm{OCH}_{2} \mathrm{CH}_{2}\right), 2.69$ (3H, s, Me), $0.94\left(2 \mathrm{H}, \mathrm{m}, \mathrm{OCH}_{2} \mathrm{CH}_{2}\right), 0.00\left(9 \mathrm{H}, \mathrm{s}, \mathrm{SiMe}_{3}\right) ; \delta_{\mathrm{C}}(100 \mathrm{MHz}$; $\left.\mathrm{CDCl}_{3}\right) 166.5(\mathrm{C}), 153.0(\mathrm{C}), 115.6(\mathrm{CH}), 94.5\left(\mathrm{CH}_{2}\right), 65.4\left(\mathrm{CH}_{2}\right), 65.1\left(\mathrm{CH}_{2}\right), 19.2(\mathrm{Me}), 18.1\left(\mathrm{CH}_{2}\right)$ and $-1.4(\mathrm{Me}) ; m / z$ (APcI) $260\left(\mathrm{MH}^{+}, 100 \%\right)$.

\footnotetext{
${ }^{3}$ Ciufolini, M. A.; Shen, Y.-C. Org. Lett. 1999, 1, 1843.
} 


\section{2-Methyl-5-(trimethylsilyl)-4-[2-(trimethylsilyl)ethoxymethoxymethyl]thiazole (11)}

A solution of $n$-butyllithium in hexanes $(2.5 \mathrm{M} ; 16.1 \mathrm{ml}, 40.3 \mathrm{mmol})$ was added dropwise over a period of 5 minutes to a solution of 2-methyl-4-[2-(trimethylsilyl)ethoxymethoxymethyl]thiazole (10) (8.69 g, $33.6 \mathrm{mmol})$ in dry tetrahydrofuran $(350 \mathrm{ml})$ at $-78{ }^{\circ} \mathrm{C}$ and the mixture was stirred for 20 minutes. A solution of chlorotrimethylsilane $(5.54 \mathrm{ml}, 43.7 \mathrm{mmol})$ in dry tetrahydrofuran $(20 \mathrm{ml})$ was added over five minutes. After stirring for 30 minutes, the reaction mixture was warmed to room temperature over the course of one hour, stirred for a further 30 minutes, quenched with water $(200 \mathrm{ml})$, concentrated in vacuo and extracted into dichloromethane $(3 \times 150 \mathrm{ml})$. The combined organic extracts were washed with brine $(50 \mathrm{ml})$, dried $\left(\mathrm{Na}_{2} \mathrm{SO}_{4}\right)$ and evaporated in vacuo. Purification by flash chromatography on silica, eluting with light petroleum-ethyl acetate (85:15), gave the title compound as a clear oil (11.0 g, 99\%) (Found: $\mathrm{MH}^{+}, 332.1530 . \mathrm{C}_{14} \mathrm{H}_{29} \mathrm{NO}_{2} \mathrm{SSi}_{2}$ requires $\left.M H, 332.1530\right) ; v_{\max }\left(\right.$ film) $/ \mathrm{cm}^{-1} 2956,2895$, $1509,1458,1378,1250,1178,1152,1105,1058,1037,939,840,760$ and $690 ; \delta_{\mathrm{H}}\left(400 \mathrm{MHz} ; \mathrm{CDCl}_{3}\right)$ $4.80\left(2 \mathrm{H}, \mathrm{s}, \mathrm{OCH}_{2} \mathrm{O}\right), 4.65\left(2 \mathrm{H}, \mathrm{s}, 4-\mathrm{CH}_{2}\right), 3.66\left(2 \mathrm{H}, \mathrm{t}, \mathrm{J} 6.8, \mathrm{OCH}_{2} \mathrm{CH}_{2}\right), 2.69(3 \mathrm{H}, \mathrm{s}, \mathrm{Me}), 0.96(2 \mathrm{H}, \mathrm{t}$, $J$ 6.8, $\left.\mathrm{OCH}_{2} \mathrm{CH}_{2}\right), 0.33\left(9 \mathrm{H}, \mathrm{s}, 5-\mathrm{SiMe}_{3}\right), 0.00\left(9 \mathrm{H}, \mathrm{s}, \mathrm{SiMe}_{3}\right) ; \delta_{\mathrm{C}}\left(100 \mathrm{MHz} ; \mathrm{CDCl}_{3}\right) 169.5(\mathrm{C}), 157.8$ (C), $130.3(\mathrm{C}), 94.7\left(\mathrm{CH}_{2}\right), 65.5\left(\mathrm{CH}_{2}\right), 65.4\left(\mathrm{CH}_{2}\right), 18.8(\mathrm{Me}), 18.1\left(\mathrm{CH}_{2}\right), 0.5(\mathrm{Me})$ and $-1.4(\mathrm{Me})$; $m / z$ (APcI) $332\left(\mathrm{MH}^{+}, 16 \%\right), 214$ (11), 184 (25), 171 (23), 146 (18), 83 (32) and 71 (100).

\section{1-[2-(2-Propenyl)oxazol-4-yl]-2-\{5-(trimethylsilyl)-4-[2-(trimethylsilyl)ethoxymethoxymethyl]-} thiazol-2-yl\}ethanone and 1-hydroxy-1-[2-(2-propenyl)oxazol-4-yl]-2-\{5-(trimethylsilyl)-4-[2(trimethylsilyl)ethoxymethoxymethyl]thiazol-2-yl\}ethene (12)

A solution of $n$-butyllithium in hexanes $(2.5 \mathrm{M} ; 0.77 \mathrm{ml}, 1.93 \mathrm{mmol})$ was added dropwise over 2 minutes to a solution of 2-methyl-5-(trimethylsilyl)-4-[2-(trimethylsilyl)ethoxymethoxymethyl]thiazole (11) $(0.61 \mathrm{~g}, 1.84 \mathrm{mmol})$ in dry tetrahydrofuran $(18 \mathrm{ml})$ at $-78{ }^{\circ} \mathrm{C}$. The mixture was stirred for 30 minutes and a solution of $N$-methoxy- $N$-methyl-2-(2-propenyl)oxazole-4-carboxamide (9) (287 mg, $1.46 \mathrm{mmol})$ in dry tetrahydrofuran $(2 \mathrm{ml})$ was added dropwise over 5 minutes. The mixture was stirred at $-78{ }^{\circ} \mathrm{C}$ for 30 minutes, warmed to room temperature over 30 minutes, stirred for a further 30 minutes and poured over ice (30 g). An aqueous solution of orthophosphoric acid (20\%; $30 \mathrm{ml})$ was added and the mixture was concentrated in vacuo and partitioned between water $(30 \mathrm{ml})$ and chloroform $(30 \mathrm{ml})$. The aqueous layer was further extracted with chloroform $(2 \times 30 \mathrm{ml})$ and the combined organic extracts were washed sequentially with an aqueous solution of orthophosphoric acid $(10 \% ; 15 \mathrm{ml})$, saturated aqueous sodium hydrogen carbonate solution $(15 \mathrm{ml})$ and brine $(15 \mathrm{ml})$, dried $\left(\mathrm{Na}_{2} \mathrm{SO}_{4}\right)$ and evaporated 
in vасио. Purification by flash chromatography on silica, eluting with light petroleum-ethyl acetate (3 : 1), gave the title compounds as an orange oil (309 mg, 45\%); $v_{\max }(\mathrm{film}) / \mathrm{cm}^{-1} 3167,3106,2954,2895$, 1651, 1546, 1448, 1249, 939, 916, 841, 766 and 733; $\delta_{\mathrm{H}}\left(400 \mathrm{MHz} ; \mathrm{CDCl}_{3}\right) 12.55(0.74 \mathrm{H}, \mathrm{bs}, \mathrm{OH})$, $8.23(0.26 \mathrm{H}, \mathrm{s}, \mathrm{OxaH}), 7.89(0.74 \mathrm{H}, \mathrm{s}, \mathrm{OxaH}), 6.52(0.74 \mathrm{H}, \mathrm{s}, \mathrm{CH}), 5.99(0.26 \mathrm{H}, \mathrm{m}, \mathrm{CHH}), 5.96$ $(0.74 \mathrm{H}, \mathrm{d}, J 1.0, \mathrm{CHH}), 5.45(0.26 \mathrm{H}, \mathrm{m}, \mathrm{CH} H), 5.39(0.74 \mathrm{H}, \mathrm{d}, J 1.0, \mathrm{CH} H), 4.78\left(1.48 \mathrm{H}, \mathrm{s}, \mathrm{OCH}_{2} \mathrm{O}\right)$, $4.77\left(0.52 \mathrm{H}, \mathrm{s}, \mathrm{OCH}_{2} \mathrm{O}\right), 4.68\left(0.52 \mathrm{H}, \mathrm{s}, \mathrm{CH}_{2}\right), 4.67\left(0.52 \mathrm{H}, \mathrm{s}, \mathrm{ArCH}_{2} \mathrm{O}\right), 4.65\left(1.46 \mathrm{H}, \mathrm{s}, \mathrm{ArCH}_{2} \mathrm{O}\right)$, $3.66\left(2 \mathrm{H}, \mathrm{m}, \mathrm{OCH}_{2} \mathrm{CH}_{2}\right), 2.15(3 \mathrm{H}, \mathrm{s}, \mathrm{Me}), 0.96\left(2 \mathrm{H}, \mathrm{m}, \mathrm{OCH}_{2} \mathrm{CH}_{2}\right), 0.34\left(9 \mathrm{H}, \mathrm{s}, 5-\mathrm{SiMe}_{3}\right), 0.00$ (6.66H, s, $\left.\mathrm{SiMe}_{3}\right),-0.02\left(2.34 \mathrm{H}, \mathrm{s}, \mathrm{SiMe}_{3}\right) ; \delta_{\mathrm{C}}\left(100 \mathrm{MHz} ; \mathrm{CDCl}_{3}\right) 189.5$ (C), 170.5 (C), 164.8 (C), 162.8 (C), 162.7 (C), 157.7 (C), 155.7 (C), 153.8 (C), 142.9 (CH), 140.5 (C), 138.1 (C), 137.2 (CH), 131.9 (C), $131.4(\mathrm{C}), 131.1(\mathrm{C}), 126.2(\mathrm{C}), 120.1\left(\mathrm{CH}_{2}\right), 119.0\left(\mathrm{CH}_{2}\right), 94.6\left(\mathrm{CH}_{2}\right), 94.5\left(\mathrm{CH}_{2}\right), 92.0(\mathrm{CH})$, $65.6\left(\mathrm{CH}_{2}\right), 65.5\left(\mathrm{CH}_{2}\right), 65.4\left(\mathrm{CH}_{2}\right), 64.7\left(\mathrm{CH}_{2}\right), 43.6\left(\mathrm{CH}_{2}\right), 19.0(\mathrm{Me}), 18.9(\mathrm{Me}), 18.1\left(\mathrm{CH}_{2}\right), 0.5(\mathrm{Me})$ and $-1.4(\mathrm{Me}) ; \mathrm{m} / z(\mathrm{APcI}) 467\left(\mathrm{MH}^{+}, 1 \%\right), 318(16), 90(100)$.

\section{1-[2-(2-Propenyl)oxazol-4-yl]-2-\{4-[2-(trimethylsilyl)ethoxymethoxymethyl]thiazol-2-yl\}ethanone}

and 1-hydroxy-1-[2-(2-propenyl)oxazol-4-yl]-2-\{4-[2-(trimethylsilyl)ethoxymethoxymethyl]thiazol-2-yl\}ethene (13)

A solution of tetrabutylammonium fluoride in tetrahydrofuran (1 M; $0.66 \mathrm{ml}, 0.66 \mathrm{mmol})$ and trifluoroacetic acid $(51 \mu \mathrm{l}, 0.66 \mathrm{mmol})$ was added to a solution of 1-[2-(2-propenyl)oxazol-4-yl]-2-\{5(trimethylsilyl)-4-[2-(trimethylsilyl)ethoxymethoxymethyl]thiazol-2-yl \}ethanone and 1-hydroxy-1-[2(2-propenyl)oxazol-4-yl]-2-\{5-(trimethylsilyl)-4-[2-(trimethylsilyl)ethoxymethoxymethyl]thiazol-2yl \}ethene (12) (309 mg, $0.66 \mathrm{mmol})$ in tetrahydrofuran $(10 \mathrm{ml})$. The mixture was stirred for 1 hour, concentrated in vacuo and partitioned between water $(30 \mathrm{ml})$ and chloroform $(30 \mathrm{ml})$. The aqueous layer was further extracted with chloroform $(2 \times 20 \mathrm{ml})$ and the combined organic extracts were dried $\left(\mathrm{Na}_{2} \mathrm{SO}_{4}\right)$ and evaporated in vacuo. Purification by flash chromatography on silica, eluting with light petroleum-ethyl acetate $(3: 1)$, gave the title compounds as an orange oil (187 mg, 78\%) (Found: $\mathrm{MH}^{+}$, 395.1456. $\mathrm{C}_{18} \mathrm{H}_{26} \mathrm{~N}_{2} \mathrm{O}_{4} \mathrm{SSi}$ requires $\left.M H, 395.1461\right) ; v_{\max }\left(\right.$ film) $/ \mathrm{cm}^{-1} 3112,2985,1694,1652,1546$, 1458, 118, 1060, 913, 860, 836, 768 and 732; $\delta_{\mathrm{H}}\left(400 \mathrm{MHz} ; \mathrm{CDCl}_{3}\right) 12.56(0.58 \mathrm{H}, \mathrm{bs}, \mathrm{OH}), 8.23$ (0.42H, s, OxaH), 7.89 (0.58H, s, OxaH), 7.19 (0.42H, s, ThzH), 6.97 (0.58H, s, ThzH), $6.51(0.58 \mathrm{H}, \mathrm{s}$, $\mathrm{CH}), 5.99(0.42 \mathrm{H}, \mathrm{m}, \mathrm{CHH}), 5.96(0.58 \mathrm{H}, \mathrm{m}, \mathrm{CHH}), 5.46(0.42 \mathrm{H}, \mathrm{d}, J$ 1.1, CHH), $5.40(0.58 \mathrm{H}, \mathrm{d}, J$ 1.4, $\mathrm{CH} H), 4.77\left(2 \mathrm{H}, \mathrm{s}, \mathrm{OCH}_{2} \mathrm{O}\right), 4.68\left(0.84 \mathrm{H}, \mathrm{s}, \mathrm{CH}_{2}\right), 4.67\left(1.16 \mathrm{H}, \mathrm{s}, \mathrm{ThzCH}_{2} \mathrm{O}\right), 4.67(0.84 \mathrm{H}, \mathrm{s}$, $\left.\mathrm{ThzCH}_{2} \mathrm{O}\right), 3.66\left(1.16 \mathrm{H}, \mathrm{t}, J\right.$ 8.0, $\left.\mathrm{OCH}_{2} \mathrm{CH}_{2}\right), 3.64\left(0.84 \mathrm{H}, \mathrm{t}, J\right.$ 8.0, $\left.\mathrm{OCH}_{2} \mathrm{CH}_{2}\right), 2.16(3 \mathrm{H}, \mathrm{s}, \mathrm{Me}), 0.94$ $\left(1.16 \mathrm{H}, \mathrm{t}, J\right.$ 8.0, $\left.\mathrm{OCH}_{2} \mathrm{CH}_{2}\right), 0.93\left(0.84 \mathrm{H}, \mathrm{t}, J\right.$ 8.0, $\left.\mathrm{OCH}_{2} \mathrm{CH}_{2}\right), 0.00\left(5.22 \mathrm{H}, \mathrm{s}, \mathrm{SiMe}_{3}\right),-0.01(3.78 \mathrm{H}, \mathrm{s}$, 
$\left.\mathrm{SiMe}_{3}\right) ; \delta_{\mathrm{C}}\left(100 \mathrm{MHz} ; \mathrm{CDCl}_{3}\right) 189.2(\mathrm{C}), 168.2(\mathrm{C}), 162.8(\mathrm{C}), 161.9$ (C), 153.6 (C), 151.7 (C), 143.6 (C), $142.8(\mathrm{CH}), 140.4(\mathrm{C}), 137.8(\mathrm{C}), 137.2(\mathrm{CH}), 131.4(\mathrm{C}), 131.0(\mathrm{C}), 120.1\left(\mathrm{CH}_{2}\right), 119.0\left(\mathrm{CH}_{2}\right)$, 117.2 $(\mathrm{CH}), 112.0(\mathrm{CH}), 94.4\left(\mathrm{CH}_{2}\right), 92.4(\mathrm{CH}), 65.5\left(\mathrm{CH}_{2}\right), 65.4\left(\mathrm{CH}_{2}\right), 65.1\left(\mathrm{CH}_{2}\right), 64.6\left(\mathrm{CH}_{2}\right), 43.7$ $\left(\mathrm{CH}_{2}\right), 19.0(\mathrm{Me})$ and $-1.4(\mathrm{Me}) ; \mathrm{m} / \mathrm{z}(\mathrm{APcI}) 395\left(\mathrm{MH}^{+}, 100 \%\right)$.

\section{1-Amino-1-[2-(2-propenyl)oxazol-4-yl]-2-\{4-[2-(trimethylsilyl)ethoxymethoxymethyl]thiazol-2- yl\}ethene (3a)}

Ammonium acetate $(53 \mathrm{mg}, 0.69 \mathrm{mmol})$ was added to a solution of 1-[2-(2-propenyl)oxazol-4-yl]-2\{4-[2-(trimethylsilyl)ethoxymethoxymethyl]thiazol-2-yl\}ethanone and 1-hydroxy-1-[2-(2propenyl)oxazol-4-yl]-2-\{4-[2-(trimethylsilyl)ethoxymethoxymethyl]thiazol-2-yl\}ethene (13) (45 mg, $0.11 \mathrm{mmol})$ in toluene $(1.5 \mathrm{ml})$ under nitrogen and the mixture was irradiated at $120{ }^{\circ} \mathrm{C}$ (initial power $100 \mathrm{~W}$ ) in a CEM Discover ${ }^{\mathrm{TM}}$ microwave synthesizer for 30 minutes. After cooling, the mixture was partitioned between water $(15 \mathrm{ml})$ and chloroform $(15 \mathrm{ml})$ and the aqueous layer further extracted with chloroform ( $2 \times 15 \mathrm{ml})$. The combined organic extracts were dried $\left(\mathrm{MgSO}_{4}\right)$ and concentrated in vacuo. Purification by flash chromatography on silica, eluting with light petroleum-ethyl acetate (4:1), gave the title compound as a brown oil (30 mg, 67\%); $v_{\max }(\mathrm{film}) / \mathrm{cm}^{-1} 3474,3156,2925,1616,1544,1463$, 1404, 1354, 1249, 1117, 1060, 917, 860, 835 and 695; $\delta_{\mathrm{H}}\left(400 \mathrm{MHz} ; \mathrm{CDCl}_{3}\right) 7.82(1 \mathrm{H}$, s OxaH), 6.84 $(1 \mathrm{H}, \mathrm{s}, \mathrm{ThzH}), 6.77\left(2 \mathrm{H}, \mathrm{bs}, \mathrm{NH}_{2}\right), 5.96(1 \mathrm{H}, \mathrm{d}, J 1.0, \mathrm{CHH}), 5.90(1 \mathrm{H}, \mathrm{s}, \mathrm{CH}), 5.39(1 \mathrm{H}, \mathrm{dd}, J$ 1.0, 0.9, $\mathrm{CH} H), 4.78\left(2 \mathrm{H}, \mathrm{s}, \mathrm{OCH}_{2} \mathrm{O}\right), 4.67\left(2 \mathrm{H}, \mathrm{s}, \mathrm{ArCH}_{2} \mathrm{O}\right), 3.66\left(2 \mathrm{H}, \mathrm{t}, J\right.$ 8.5, $\left.\mathrm{OCH}_{2} \mathrm{CH}_{2}\right), 2.16(3 \mathrm{H}, \mathrm{d}, J 0.9$, $\mathrm{Me}), 0.94\left(2 \mathrm{H}, \mathrm{t}, J\right.$ 8.5, $\left.\mathrm{OCH}_{2} \mathrm{CH}_{2}\right), 0.00\left(9 \mathrm{H}, \mathrm{s}, \mathrm{SiMe}_{3}\right) ; \delta_{\mathrm{C}}\left(100 \mathrm{MHz} ; \mathrm{CDCl}_{3}\right) 168.3$ (C), 162.6 (C), $152.9(\mathrm{C}), 139.7(\mathrm{C}), 139.0(\mathrm{C}), 134.5(\mathrm{CH}), 131.4(\mathrm{C}) 118.9\left(\mathrm{CH}_{2}\right), 110.6(\mathrm{CH}), 94.3\left(\mathrm{CH}_{2}\right), 88.1(\mathrm{CH})$, $65.3\left(\mathrm{CH}_{2}\right), 65.3\left(\mathrm{CH}_{2}\right), 19.0(\mathrm{Me}), 18.1\left(\mathrm{CH}_{2}\right)$ and $-1.4(\mathrm{Me}) ; \mathrm{m} / z(\mathrm{APcI}) 394\left(\mathrm{MH}^{+}, 1 \%\right), 311(10)$, 241 (10), 97 (29), 83 (42) and 71 (100).

Methyl 2-[2-(2-propenyl)oxazol-4-yl]-3-\{4-[2-(trimethylsilyl)ethoxymethoxymethyl]thiazol-2-yl\}pyridine-6-carboxylate (2a)

A solution of methyl 2-oxo-4-(trimethylsilyl)-3-butynoate (4) $)^{4}(122 \mathrm{mg}, 0.66 \mathrm{mmol})$ in methanol (1 ml) was added to a solution of 1-amino-1-[2-(2-propenyl)oxazol-4-yl]-2-\{4-[2-(trimethylsilyl)ethoxymethoxymethyl]thiazol-2-yl\}ethene (3a) $(129 \mathrm{mg}, 0.33 \mathrm{mmol})$ in methanol $(5 \mathrm{ml})$ and the mixture was stirred overnight and concentrated in vacuo. The crude residue was dissolved in tetrahydrofuran (5 ml)

\footnotetext{
${ }^{4}$ Bagley, M. C.; Brace, C.; Dale, J. W.; Ohnesorge, M.; Phillips, N. G.; Xiong, X.; Bower, J. J. Chem. Soc., Perkin Trans. 1 2002, 1663.
} 
and a solution of tetrabutylammonium fluoride in tetrahydrofuran (1 M; $0.66 \mathrm{ml}, 0.66 \mathrm{mmol})$ and trifluoroacetic acid $(51 \mu 1,0.66 \mathrm{mmol})$ was added. The mixture was stirred for 1 hour at room temperature, evaporated in vacuo and partitioned between water $(50 \mathrm{ml})$ and chloroform $(50 \mathrm{ml})$. The aqueous layer was further extracted with chloroform $(2 \times 30 \mathrm{ml})$ and the combined organic extracts were dried $\left(\mathrm{MgSO}_{4}\right)$ and concentrated in vacuo. Purification by flash chromatography on silica, eluting with light petroleum-ethyl acetate (4:1), gave the title compound as a brown oil (80 mg, 50\%) (Found: $\mathrm{MH}^{+}, 488.1664 . \mathrm{C}_{23} \mathrm{H}_{29} \mathrm{~N}_{3} \mathrm{O}_{2} \mathrm{SSi}$ requires $\left.M H, 488.1675\right) ; v_{\max }\left(\right.$ film)/cm $\mathrm{cm}^{-1} 3096,2956,2924,2889$, $1727,1579,1538,1440,1354,1322,1278,1249,1193,1139,1111,1060,1006,916,860,837,760$

and 733; $\delta_{\mathrm{H}}\left(400 \mathrm{MHz} ; \mathrm{CDCl}_{3}\right) 8.27(1 \mathrm{H}, \mathrm{d}, J$ 8.8, 4-H), $8.15(1 \mathrm{H}, \mathrm{d}, J$ 8.8, 5-H), $7.90(1 \mathrm{H}, \mathrm{s}, \mathrm{OxaH})$, $7.37(1 \mathrm{H}, \mathrm{s}, \mathrm{ThzH}), 5.90(1 \mathrm{H}, \mathrm{m}, \mathrm{CHH}), 5.35(1 \mathrm{H}, \mathrm{m}, \mathrm{CH}), 4.79\left(2 \mathrm{H}, \mathrm{s}, \mathrm{OCH}_{2} \mathrm{O}\right), 4.76(2 \mathrm{H}, \mathrm{s}$, ThzCH$\left.H_{2} \mathrm{O}\right), 4.00(3 \mathrm{H}, \mathrm{s}, \mathrm{OMe}), 3.67\left(2 \mathrm{H}, \mathrm{t}, J\right.$ 8.4, $\left.\mathrm{OCH}_{2} \mathrm{CH}_{2}\right), 2.06(3 \mathrm{H}, \mathrm{s}, \mathrm{Me}), 0.95(2 \mathrm{H}, \mathrm{t}, J$ 8.4, $\left.\mathrm{OCH}_{2} \mathrm{CH}_{2}\right), 0.00\left(9 \mathrm{H}, \mathrm{s}, \mathrm{SiMe}_{3}\right) ; \delta_{\mathrm{C}}\left(100 \mathrm{MHz} ; \mathrm{CDCl}_{3}\right) 165.1$ (C), 163.6 (C), 162.3 (C), 154.3 (C), 148.9 (C), $148.0(\mathrm{C}), 139.5(\mathrm{CH}), 139.2$ (C), 138.7 (CH), 131.9 (C), 131.4 (C), 123.8 (CH), 118.6 $\left(\mathrm{CH}_{2}\right), 94.4\left(\mathrm{CH}_{2}\right), 65.4\left(\mathrm{CH}_{2}\right), 65.0\left(\mathrm{CH}_{2}\right), 53.1(\mathrm{Me}), 19.0(\mathrm{Me}), 18.1\left(\mathrm{CH}_{2}\right)$ and $-1.1(\mathrm{Me})$; one quaternary carbon not observed; $m / z$ (APcI) $488\left(\mathrm{MH}^{+}, 77 \%\right), 408$ (47), 356 (9), 348 (15), 258 (20), 109 (27), 83 (100).

\section{Methyl 2-(2-acetyloxazol-4-yl)-3-\{4-[2-(trimethylsilyl)ethoxymethoxymethyl]thiazol-2-yl\}pyrid- ine-6-carboxylate (14)}

A solution of osmium(VIII) tetroxide $(4.0 \mathrm{mg}, 0.016 \mathrm{mmol})$ in acetonitrile $(0.2 \mathrm{ml})^{5}$ was added to a solution of methyl 2-[2-(2-propenyl)oxazol-4-yl]-3-\{4-[2-(trimethylsilyl)ethoxymethoxymethyl]thiazol-2-yl pyridine-6-carboxylate (2a) $(80 \mathrm{mg}, 0.16 \mathrm{mmol})$ in dioxane-water (1:1) (20 ml). After stirring for 10 minutes, sodium periodate $(70 \mathrm{mg}, 0.33 \mathrm{mmol})$ was added, the mixture was stirred for a further 12 hours and partitioned between water $(30 \mathrm{ml})$ and dichloromethane $(30 \mathrm{ml})$. The aqueous layer was further extracted with dichloromethane $(30 \mathrm{ml})$ and the combined organic extracts were washed with a saturated aqueous solution of sodium metabisulfite $(30 \mathrm{ml})$, dried $\left(\mathrm{Na}_{2} \mathrm{SO}_{4}\right)$ and concentrated in vacuo. Purification by flash chromatography on silica, eluting with light petroleumethyl acetate (1 : 3), gave the title compound as a brown oil (39 mg, 50\%) (Found: $\mathrm{MH}^{+}, 490.1464$. $\mathrm{C}_{22} \mathrm{H}_{27} \mathrm{~N}_{3} \mathrm{O}_{6} \mathrm{SSi}$ requires $\left.M H, 490.1468\right) ; v_{\max }\left(\right.$ film) $/ \mathrm{cm}^{-1} 3116,2953,1728,1709,1527,1441,1370$, $1320,1288,1248,1224,1193,1133,1110,1059,860,837$ and $764 ; \delta_{\mathrm{H}}\left(400 \mathrm{MHz}\right.$; $\mathrm{d}_{4}$-methanol) 8.61 $(1 \mathrm{H}, \mathrm{s}, \mathrm{OxaH}), 8.28(1 \mathrm{H}, \mathrm{d}, J$ 8.0, 4-H), $8.20(1 \mathrm{H}, \mathrm{d}, J$ 8.0, 5-H), $7.64(1 \mathrm{H}, \mathrm{s}, \mathrm{ThzH}), 4.72(2 \mathrm{H}, \mathrm{s}$, 
$\left.\mathrm{OCH}_{2} \mathrm{O}\right), 4.68\left(2 \mathrm{H}, \mathrm{s}, \mathrm{ArCH}_{2} \mathrm{O}\right), 3.96(3 \mathrm{H}, \mathrm{s}, \mathrm{OMe}), 3.65\left(2 \mathrm{H}, \mathrm{t}, J\right.$ 8.1, $\left.\mathrm{OCH}_{2} \mathrm{CH}_{2}\right), 2.39(3 \mathrm{H}, \mathrm{s}, \mathrm{Me})$, $0.92\left(2 \mathrm{H}, \mathrm{t}, J\right.$ 8.1, $\left.\mathrm{OCH}_{2} \mathrm{CH}_{2}\right), 0.00\left(9 \mathrm{H}, \mathrm{s}, \mathrm{SiMe}_{3}\right) ; \delta_{\mathrm{C}}\left(100 \mathrm{MHz} ; \mathrm{d}_{4}\right.$-methanol) 185.3 (C), 173.7 (C), 164.8 (C), 164.1 (C), 157.0 (C), 154.1 (C), 147.9 (C), 142.7 (CH), 140.4 (C), 140.1 (CH), 131.3 (C), $124.0(\mathrm{CH}), 119.3(\mathrm{CH}), 94.0\left(\mathrm{CH}_{2}\right), 65.0\left(\mathrm{CH}_{2}\right), 64.2\left(\mathrm{CH}_{2}\right), 52.2(\mathrm{Me}), 25.3(\mathrm{Me}), 17.5\left(\mathrm{CH}_{2}\right)$ and 2.7 (Me); m/z (APcI) $490\left(\mathrm{MH}^{+}, 100 \%\right), 328$ (33), 124 (100).

\section{Formation of lithium enolate of $S$-ethyl thioacetate}

A solution of $n$-butyllithium in hexanes $(2.5 \mathrm{M} ; 6.24 \mathrm{ml}, 15.6 \mathrm{mmol})$ was added to a stirred solution of diisopropylamine $(2.20 \mathrm{ml}, 15.6 \mathrm{mmol})$ in tetrahydrofuran $(15.7 \mathrm{ml})$ at $0{ }^{\circ} \mathrm{C}$. The mixture was stirred for $10 \mathrm{~min}$ and cooled to $-78{ }^{\circ} \mathrm{C}$. Freshly distilled $S$-ethyl thioacetate $(0.83 \mathrm{ml}, 7.8 \mathrm{mmol})$ was added and the solution was stirred for $30 \mathrm{~min}$.

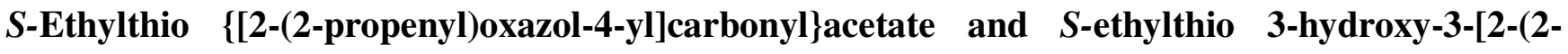 propenyl)oxazol-4-yl]propenoate (15)}

Ethyl chloroformate $(0.54 \mathrm{ml}, 5.7 \mathrm{mmol})$ was added dropwise to a stirred solution of 2-(2propenyl)oxazole-4-carboxylic acid $(8)(800 \mathrm{mg}, 5.22 \mathrm{mmol})$ and triethylamine $(0.80 \mathrm{ml}, 5.7 \mathrm{mmol})$ in dry tetrahydrofuran $(11 \mathrm{ml})$ at $0{ }^{\circ} \mathrm{C}$. After stirring for 30 minutes, the mixture was filtered, cooled to $-78{ }^{\circ} \mathrm{C}$ and a solution of the lithium enolate of $S$-ethyl thioacetate was added dropwise. The mixture was stirred at $-78{ }^{\circ} \mathrm{C}$ for $30 \mathrm{~min}$ and partitioned between saturated aqueous ammonium chloride solution $(60 \mathrm{ml})$ and ethyl acetate $(60 \mathrm{ml})$. The organic extract was washed with brine $(40 \mathrm{ml})$, dried $\left(\mathrm{Na}_{2} \mathrm{SO}_{4}\right)$ and evaporated in vacuo. Purification by flash chromatography on silica, eluting with dichloromethane, gave the title compounds as a pale yellow oil (0.94 g, 75\%) (Found: $\mathrm{MH}^{+}, 240.0688$. $\mathrm{C}_{11} \mathrm{H}_{13} \mathrm{NO}_{3} \mathrm{~S}$ requires $\left.\mathrm{MH}^{+}, 240.0689\right) ; v_{\max }(\mathrm{KBr}) / \mathrm{cm}^{-1}$ 2966, 2925, 1704, 1648, 1589, 1538, 1452 , 1408, 1330, 1246, 1120, 1080, 775, 722; $\delta_{\mathrm{H}}\left(400 \mathrm{MHz} ; \mathrm{CDCl}_{3}\right) 12.54(0.65 \mathrm{H}, \mathrm{s}, \mathrm{OH}), 8.15(0.35 \mathrm{H}, \mathrm{s}$, OxaH), $7.93(0.65 \mathrm{H}, \mathrm{s}, \mathrm{OxaH}), 6.20(0.65 \mathrm{H}, \mathrm{s}, \mathrm{CH}), 5.94(0.35 \mathrm{H}, \mathrm{s}, \mathrm{CHH}), 5.91(0.65 \mathrm{H}, \mathrm{s}, \mathrm{CHH}), 5.40$ $(0.35 \mathrm{H}, \mathrm{s}, \mathrm{CH} H), 5.37(0.65 \mathrm{H}, \mathrm{s}, \mathrm{CH} H), 4.10\left(0.70 \mathrm{H}, \mathrm{s}, \mathrm{CH}_{2}\right), 2.90\left(1.30 \mathrm{H}, \mathrm{q}, J\right.$ 7.4, $\left.\mathrm{CH}_{2} \mathrm{Me}\right), 2.86$ $\left(0.70 \mathrm{H}, \mathrm{q}, J\right.$ 7.4, $\left.\mathrm{CH}_{2} \mathrm{Me}\right), 2.09$ (3H, s, Me), $1.24\left(1.95 \mathrm{H}, \mathrm{t}, J\right.$ 7.4, $\left.\mathrm{CH}_{2} \mathrm{Me}\right), 1.19(1.05 \mathrm{H}, \mathrm{t}, J$ 7.4, $\left.\mathrm{CH}_{2} \mathrm{Me}\right) ; \delta_{\mathrm{C}}\left(100 \mathrm{MHz}, \mathrm{CDCl}_{3}\right) 195.5$ (C), $192.0(\mathrm{C}), 186.9$ (C), $163.0(\mathrm{C}), 162.8$ (C), 161.0 (C), 142.8 (CH), $140.7(\mathrm{C}), 139.7(\mathrm{CH}), 136.6(\mathrm{C}), 131.2(\mathrm{C}), 131.0(\mathrm{C}), 120.1\left(\mathrm{CH}_{2}\right), 119.6\left(\mathrm{CH}_{2}\right), 98.3(\mathrm{CH})$, $54.5\left(\mathrm{CH}_{2}\right), 24.0\left(\mathrm{CH}_{2}\right), 22.9\left(\mathrm{CH}_{2}\right), 18.91(\mathrm{Me}), 18.88(\mathrm{Me}), 14.8(\mathrm{Me}), 14.5(\mathrm{Me}) ; \mathrm{m} / z(\mathrm{APcI}) 240$ $\left(\mathrm{MH}^{+}, 100 \%\right), 210(44)$.

\footnotetext{
${ }^{5}$ From a $0.079 \mathrm{M}$ stock solution of osmium(VIII) tetroxide $(250 \mathrm{mg}, 0.98 \mathrm{mmol})$ in acetonitrile $(12.5 \mathrm{ml})$.
} 
(S)-N-\{3-Hydroxy-3-[2-(2-propenyl)oxazol-4-yl]propenoyl\}-O-tert-butylserine methyl ester and (S)- $N$-\{3-oxo-3-[2-(2-propenyl)oxazol-4-yl]propanoyl\}-O-tert-butylserine methyl ester (16)

A solution of $S$-ethylthio \{[2-(2-propenyl)oxazol-4-yl]carbonyl $\}$ acetate and $S$-ethylthio 3-hydroxy-3[2-(2-propenyl)oxazol-4-yl]propenoate (15) $(274 \mathrm{mg}, 1.14 \mathrm{mmol})$ in dry dichloromethane $(5 \mathrm{ml})$ was added to a stirred solution of triethylamine $(0.32 \mathrm{ml}, 2.29 \mathrm{mmol})$ and $O$-tert-butyl-L-serine methyl ester hydrochloride (242 mg, $1.14 \mathrm{mmol}$ ) in dry dichloromethane (15 ml). Copper(I) iodide (485 mg, 2.29 mmol) was added, the mixture was stirred at room temperature overnight, partitioned between dichloromethane $(5 \mathrm{ml})$ and dilute hydrochloric acid $(1 \mathrm{~N} ; 5 \mathrm{ml})$ and filtered. The organic extract was washed sequentially with dilute hydrochloric acid $(1 \mathrm{~N} ; 10 \mathrm{ml})$, saturated aqueous sodium hydrogen carbonate solution $(10 \mathrm{ml})$ and brine $(10 \mathrm{ml})$, dried $\left(\mathrm{Na}_{2} \mathrm{SO}_{4}\right)$ and evaporated in vacuo. Purification by flash column chromatography on silica gel, eluting with light petroleum-ethyl acetate (4:1), gave the title compounds as a pale yellow oil $\left(0.33 \mathrm{~g}, 83 \%\right.$ ) (Found: $\mathrm{MH}^{+}, 353.1701 . \mathrm{C}_{17} \mathrm{H}_{24} \mathrm{~N}_{2} \mathrm{O}_{6}$ requires $\mathrm{MH}^{+}$, 353.1707); $[\alpha]_{\mathrm{D}}^{24}+41.7\left(c 1.1, \mathrm{CHCl}_{3}\right) ; v_{\max }(\mathrm{KBr}) / \mathrm{cm}^{-1} 3366,2971,1750,1694,1661,1609,1549$, 1438, 1364, 1248, 1204, 1093, 1054, 1020, 915, 812, 780, 737; $\delta_{\mathrm{H}}\left(400 \mathrm{MHz} ; \mathrm{CDCl}_{3}\right) 13.36(0.36 \mathrm{H}, \mathrm{s}$, $\mathrm{OH}), 8.24(0.64 \mathrm{H}, \mathrm{s}, \mathrm{OxaH}), 7.87(0.36 \mathrm{H}, \mathrm{s}, \mathrm{OxaH}), 7.72(0.64 \mathrm{H}, \mathrm{d}, J$ 8.2, NH), $6.42(0.36 \mathrm{H}, \mathrm{d}, J$ 8.5, $\mathrm{NH}), 5.95(0.64 \mathrm{H}, \mathrm{s}, 2$ '-H), $5.90(0.36 \mathrm{H}, \mathrm{s}, 2$ 2"-H), $5.79(0.36 \mathrm{H}, \mathrm{s}, \mathrm{CH}), 5.42(0.64 \mathrm{H}, \mathrm{s}, 2$ 2"-H), 5.35 (0.36H, s, 2"-H), $4.71(0.36 \mathrm{H}, \mathrm{m}, \alpha-\mathrm{CH}), 4.66(0.64 \mathrm{H}, \mathrm{m}, \alpha-\mathrm{CH}), 3.93(0.64 \mathrm{H}, \mathrm{d}, J$ 15.6, CHH), 3.89 $(0.64 \mathrm{H}, \mathrm{d}, J$ 15.6, CHH), $3.78(0.36 \mathrm{H}, \mathrm{dd}, J$ 8.9, 2.9, $\beta-\mathrm{CH}), 3.74(0.64 \mathrm{H}, \mathrm{dd}, J$ 9.1, 3.0, $\beta-\mathrm{CH})$, $3.68(1.08 \mathrm{H}, \mathrm{s}, \mathrm{OMe}), 3.65(1.92 \mathrm{H}, \mathrm{s}, \mathrm{OMe}), 3.54(0.36 \mathrm{H}, \mathrm{dd}, J$ 8.9, 3.2, $\beta-\mathrm{CH} H), 3.49(0.64 \mathrm{H}, \mathrm{dd}, J$ 9.1, 3.2, $\beta-\mathrm{CH} H), 2.11(1.92 \mathrm{H}, \mathrm{s}, \mathrm{Me}), 2.08(1.08 \mathrm{H}, \mathrm{s}, \mathrm{Me}), 1.06\left(1.08 \mathrm{H}, \mathrm{s}, \mathrm{CMe}_{3}\right), 1.04(1.92 \mathrm{H}, \mathrm{s}$, $\left.\mathrm{CMe}_{3}\right) ; \delta_{\mathrm{C}}\left(100 \mathrm{MHz}, \mathrm{CDCl}_{3}\right) 188.8(\mathrm{C}), 171.3(\mathrm{C}), 170.8(\mathrm{C}), 170.6$ (C), $165.2(\mathrm{C}), 162.8(\mathrm{C}), 162.7$ (C), $162.2(\mathrm{C}), 143.2(\mathrm{CH}), 140.7(\mathrm{C}), 138.1(\mathrm{CH}), 137.4(\mathrm{C}), 131.2(\mathrm{C}), 130.9(\mathrm{C}), 120.2\left(\mathrm{CH}_{2}\right), 119.2$ $\left(\mathrm{CH}_{2}\right), 89.9(\mathrm{CH}), 73.4(\mathrm{C}), 73.3(\mathrm{C}), 61.9\left(\mathrm{CH}_{2}\right), 61.8\left(\mathrm{CH}_{2}\right), 53.1(\mathrm{CH}), 52.4(\mathrm{Me}), 52.3(\mathrm{Me}), 52.3$ (CH), $47.0\left(\mathrm{CH}_{2}\right), 27.2(\mathrm{Me}), 27.2(\mathrm{Me}), 18.9(\mathrm{Me}), 18.8(\mathrm{Me}) ; \mathrm{m} / z(\mathrm{APcI}) 353\left(\mathrm{MH}^{+}, 44 \%\right), 297$ (100), 279 (12), 120 (37).

\section{(S)-N-\{3-Amino-3-[2-(2-propenyl)oxazol-4-yl]propenoyl\}-O-tert-butylserine methyl ester (3b)}

Ammonium acetate $(293 \mathrm{mg}, 3.8 \mathrm{mmol})$ was added to a solution of $(S)-N$-\{3-hydroxy-3-[2-(2propenyl)oxazol-4-yl]propenoyl\}-O-tert-butylserine methyl ester and $(S)-N$-\{3-oxo-3-[2-(2propenyl)oxazol-4-yl]propanoyl\}-O-tert-butylserine methyl ester (16) $(269 \mathrm{mg}, 0.76 \mathrm{mmol})$ in dry methanol (15 ml) under nitrogen and the reaction heated at reflux overnight. After cooling to room 
temperature, the mixture was evaporated in vасио. The residue was partitioned between ethyl acetate $(30 \mathrm{ml})$ and water $(30 \mathrm{ml})$ and the aqueous layer further extracted with ethyl acetate $(30 \mathrm{ml})$. The combined organic extracts were washed sequentially with saturated aqueous sodium hydrogen carbonate solution $(30 \mathrm{ml})$ and brine $(30 \mathrm{ml})$, dried $\left(\mathrm{Na}_{2} \mathrm{SO}_{4}\right)$ and evaporated in vacuo. Purification by flash chromatography on silica, eluting with light petroleum-ethyl acetate (1:1), gave the title compound as a pale yellow oil (214 mg, 80\%) (Found: $\mathrm{MH}^{+}$, 352.1871. $\mathrm{C}_{17} \mathrm{H}_{25} \mathrm{~N}_{3} \mathrm{O}_{5}$ requires $M H^{+}$, 352.1867); $[\alpha]_{\mathrm{D}}^{24}+51.2\left(c 1.0, \mathrm{CHCl}_{3}\right) ; v_{\max }(\mathrm{KBr}) / \mathrm{cm}^{-1} 3452,3324,2974,1748,1644,1598,1540$, 1363, 1198, 1098, 1050, 1021, 976, 913, 778; $\delta_{\mathrm{H}}\left(400 \mathrm{MHz} ; \mathrm{CDCl}_{3}\right) 7.79(1 \mathrm{H}, \mathrm{s}, \mathrm{OxaH}), 6.82(2 \mathrm{H}, \mathrm{bs}$, $\left.\mathrm{NH}_{2}\right), 5.90(1 \mathrm{H}, \mathrm{s}, \mathrm{CHH}), 5.89(1 \mathrm{H}, \mathrm{d}, J$ 8.5, NH), $5.36(1 \mathrm{H}, \mathrm{s}, \mathrm{CH} H), 5.00(1 \mathrm{H}, \mathrm{s}, \mathrm{CH}), 4.72(1 \mathrm{H}, \mathrm{m}$, $\alpha-\mathrm{CH}), 3.78(1 \mathrm{H}, \mathrm{dd}, J$ 8.9, 2.9, $\beta-\mathrm{CH}), 3.68(3 \mathrm{H}, \mathrm{s}, \mathrm{OMe}), 3.51(1 \mathrm{H}, \mathrm{dd}, J$ 8.9, 3.2, $\beta-\mathrm{CH} H), 2.10$ (3H, s, Me), $1.08\left(9 \mathrm{H}, \mathrm{s}, \mathrm{CMe}_{3}\right) ; \delta_{\mathrm{C}}\left(100 \mathrm{MHz}, \mathrm{CDCl}_{3}\right) 171.8$ (C), 169.8 (C), $162.6(\mathrm{C}), 147.8$ (C), $138.6(\mathrm{C}), 135.5(\mathrm{CH}), 131.3(\mathrm{C}), 119.1\left(\mathrm{CH}_{2}\right), 84.2(\mathrm{CH}), 73.4(\mathrm{C}), 62.4\left(\mathrm{CH}_{2}\right), 52.34(\mathrm{CH}), 52.30$ (Me), $27.3(\mathrm{Me}), 19.0(\mathrm{Me}) ; \mathrm{m} / z$ (APcI) 353 (100\%), $352\left(\mathrm{MH}^{+}, 67\right)$.

\section{(S)-O-tert-Butyl- $N$-(\{2-[2-(2-propenyl)oxazol-4-yl]-6-methoxycarbonylpyridin-3-yl\}carbonyl)-} serine methyl ester (2b)

A solution of (S)- $N$-\{3-amino-3-[2-(2-propenyl)oxazol-4-yl]propenoyl $\}$-O-tert-butylserine methyl ester (3b) $(88 \mathrm{mg}, 0.25 \mathrm{mmol})$ and 4-(trimethylsilyl)-2-oxobut-3-ynoate ${ }^{6}(61 \mathrm{mg}, 0.33 \mathrm{mmol})$ in methanol $(10 \mathrm{ml})$ was stirred at room temperature for $24 \mathrm{~h}$ and evaporated in vacuo. Purification by flash chromatography on silica, eluting with light petroleum-ethyl acetate (1:2), gave the title compound as a pale yellow oil (104 mg, 93\%) (Found: $\mathrm{MH}^{+}, 446.1925 . \mathrm{C}_{22} \mathrm{H}_{27} \mathrm{~N}_{3} \mathrm{O}_{7}$ requires $M H^{+}, 446.1927$ ); $[\alpha]_{\mathrm{D}}{ }^{29}$ +12.0 (c 1.8, $\left.\mathrm{CHCl}_{3}\right) ; v_{\max }(\mathrm{KBr}) / \mathrm{cm}^{-1} 2966,1751,1670,1540,1436,1364,1323,1262,1099,801,760$; $\delta_{\mathrm{H}}\left(400 \mathrm{MHz} ; \mathrm{CDCl}_{3}\right) 8.18(1 \mathrm{H}, \mathrm{s}, \mathrm{OxaH}), 8.05(1 \mathrm{H}, \mathrm{d}, J$ 8.0, PyH), $8.01(1 \mathrm{H}, \mathrm{d}, J$ 8.0, PyH), $7.00(1 \mathrm{H}$, d, J 8.0, NH), $5.93(1 \mathrm{H}, \mathrm{s}, \mathrm{CHH}), 5.35(1 \mathrm{H}, \mathrm{s}, \mathrm{CH} H), 4.85(1 \mathrm{H}, \mathrm{m}, \alpha-\mathrm{CH}), 3.95\left(3 \mathrm{H}, \mathrm{s}, \mathrm{PyCO}_{2} \mathrm{Me}\right)$, $3.80(1 \mathrm{H}, \mathrm{dd}, J$ 9.1, 3.0, $\beta-\mathrm{CHH}), 3.69$ (3H, s, OMe), 3.57 (1H, dd, $J$ 9.1, 3.2, $\beta-\mathrm{CH} H), 2.10(3 \mathrm{H}, \mathrm{s}$, Me), $1.01\left(9 \mathrm{H}, \mathrm{s}, \mathrm{CMe}_{3}\right) ; \delta_{\mathrm{C}}\left(100 \mathrm{MHz}, \mathrm{CDCl}_{3}\right) 170.5$ (C), 167.0 (C), 165.0 (C), 162.6 (C), 148.5 (C), $147.5(\mathrm{C}), 139.5(\mathrm{C}), 139.0(\mathrm{CH}), 138.1(\mathrm{CH}), 133.3(\mathrm{C}), 131.4(\mathrm{C}), 123.6(\mathrm{CH}), 119.0\left(\mathrm{CH}_{2}\right), 73.6(\mathrm{C})$, $61.8\left(\mathrm{CH}_{2}\right), 53.5(\mathrm{CH}), 53.1(\mathrm{Me}), 52.5(\mathrm{Me}), 27.2(\mathrm{Me}), 19.0(\mathrm{Me}) ; \mathrm{m} / z(\mathrm{APcI}) 446\left(\mathrm{MH}^{+}, 100 \%\right)$. 

ester (17)

A solution of (S)-O-tert-butyl- $N$-(\{2-[2-(2-propenyl)oxazol-4-yl]-6-methoxycarbonylpyridin-3yl \}carbonyl)serine methyl ester $(\mathbf{2 b})(60 \mathrm{mg}, 0.14 \mathrm{mmol})$ in trifluoroacetic acid-dichloromethane $(1: 1)$ $(20 \mathrm{ml})$ was stirred at room temperature for $20 \mathrm{~min}$ and evaporated in vacuo. Purification by flash column chromatography on silica, eluting with ethyl acetate, gave the title compound as colourless crystals (50 mg, 96\%), mp 74-76 ${ }^{\circ} \mathrm{C}$ (aqueous ethanol) (Found: $\mathrm{MH}^{+}$, 390.1301. $\mathrm{C}_{18} \mathrm{H}_{19} \mathrm{~N}_{3} \mathrm{O}_{7}$ requires $\left.M H^{+}, 390.1296\right) ;[\alpha]_{\mathrm{D}}{ }^{25}-8.8\left(c \quad 0.5, \mathrm{CHCl}_{3}\right) ; v_{\max }(\mathrm{KBr}) / \mathrm{cm}^{-1} 3439,2954,1734,1654,1542,1438$, 1323, 1293, 1234, 1174, 1140, 760; $\delta_{\mathrm{H}}\left(400 \mathrm{MHz} ; \mathrm{CDCl}_{3}\right) 8.22(1 \mathrm{H}, \mathrm{s}, \mathrm{OxaH}), 7.89(1 \mathrm{H}, \mathrm{d}, J$ 7.9, PyH), $7.84(1 \mathrm{H}, \mathrm{d}, J$ 7.9, PyH), $7.50(1 \mathrm{H}, \mathrm{d}, J$ 7.1, NH), $5.89(1 \mathrm{H}, \mathrm{s}, \mathrm{CHH}), 5.35(1 \mathrm{H}, \mathrm{s}, \mathrm{CH}), 4.70(1 \mathrm{H}, \mathrm{m}$, $\alpha-\mathrm{CH}), 4.32(1 \mathrm{H}, \mathrm{bs}, \mathrm{OH}), 4.01(1 \mathrm{H}, \mathrm{dd}, J$ 8.2, 3.1, $\beta-\mathrm{CHH}), 3.94(1 \mathrm{H}, \mathrm{dd}, J$ 8.2, 3.7, $\beta-\mathrm{CH} H), 3.91$ $\left(3 \mathrm{H}, \mathrm{s}, \mathrm{PyCO}_{2} \mathrm{Me}\right), 3.68(3 \mathrm{H}, \mathrm{s}, \mathrm{OMe}), 2.05(3 \mathrm{H}, \mathrm{s}, \mathrm{Me}) ; \delta_{\mathrm{C}}\left(100 \mathrm{MHz}, \mathrm{CDCl}_{3}\right) 170.5(\mathrm{C}), 167.6(\mathrm{C})$, 164.9 (C), 162.8 (C), 148.0 (C), 147.2 (C), 142.2 (C), 139.7 (CH), 137.8 (CH), 132.8 (C), 131.0 (C), $123.5(\mathrm{CH}), 119.9\left(\mathrm{CH}_{2}\right), 62.2\left(\mathrm{CH}_{2}\right), 55.6(\mathrm{CH}), 53.2(\mathrm{Me}), 52.8(\mathrm{Me}), 18.9(\mathrm{Me}) ; \mathrm{m} / z(\mathrm{APcI}) 390$ $\left(\mathrm{MH}^{+}, 100 \%\right)$.

\section{Methyl 2-(2-acetyloxazol-4-yl)-3-[4-(methoxycarbonyl)thiazol-2-yl]pyridine-6-carboxylate} (dimethyl sulfomycinamate) (1)

A solution of $(S)-N$-(\{2-[2-(2-propenyl)oxazol-4-yl]-6-methoxycarbonylpyridin-3-yl $\}$ carbonyl)serine methyl ester (17) (41 mg, $0.10 \mathrm{mmol})$ and Burgess reagent $(28 \mathrm{mg}, 0.11 \mathrm{mmol})$ in dry tetrahydrofuran $(5 \mathrm{ml})$ was stirred at $70{ }^{\circ} \mathrm{C}$ for $1 \mathrm{~h}$ and evaporated in vacuo. Purification by flash chromatography on silica, eluting with light petroleum-ethyl acetate (1:2), gave methyl 2-[2-(2-propenyl)oxazol-4-yl]-3-(4methoxycarbonyl-2-oxazolin-2-yl)pyridine-6-carboxylate (18) as a pale yellow oil (24 mg, 63\%). A solution of this oil in methanol-triethylamine (2:1) (3 ml) was saturated with hydrogen sulfide, stirred at room temperature for $3.5 \mathrm{~h}$ and evaporated in vacuo. Purification by flash chromatography on silica, eluting with diethyl ether-acetone (5:1), gave $\mathrm{N}-(\{2-[2-(2-$ propenyl)oxazol-4-yl]-6methoxycarbonylpyridin-3-yl\}thiocarbonyl)serine methyl ester (19) as a pale yellow oil (17 mg, 71\%). A solution of thioamide $19(33 \mathrm{mg}, 0.08 \mathrm{mmol})$ and Burgess reagent (24 mg, $0.10 \mathrm{mmol})$ in dry tetrahydrofuran $(5 \mathrm{ml})$ was stirred at $70{ }^{\circ} \mathrm{C}$ for $30 \mathrm{~min}$ and evaporated in vacuo. Purification by flash chromatography on silica, eluting with ethyl acetate, gave methyl 2-[2-(2-propenyl)oxazol-4-yl]-3-(4-

\footnotetext{
${ }^{6}$ Bagley, M. C.; Brace, C.; Dale, J. W.; Ohnesorge, M.; Phillips, N. G.; Xiong, X.; Bower, J. J. Chem. Soc., Perkin Trans. 1 2002, 1663.
} 
methoxycarbonyl-2-thiazolin-2-yl)pyridine-6-carboxylate (20) as a pale yellow oil (27 $\mathrm{mg}, 87 \%)$. A mixture of thiazoline $\mathbf{2 0}$ and activated manganese(IV) oxide (121 mg, $1.39 \mathrm{mmol})$ in dichloromethane $(3 \mathrm{ml})$ was irradiated at $100{ }^{\circ} \mathrm{C}$ (initial power $300 \mathrm{~W}$ ) for $150 \mathrm{~min}$ in a sealed pressure-rated reaction tube $(10 \mathrm{ml})$ using a CEM Discover ${ }^{\mathrm{TM}}$ Microwave Synthesizer. The mixture was cooled rapidly to room temperature in a flow of compressed air for $5 \mathrm{~min}$, filtered through Celite ${ }^{\circledR}$ washing with dichloromethane $(2 \times 10 \mathrm{ml})$ and evaporated in vacuo. Purification by flash chromatography on silica, eluting with light petroleum-ethyl acetate (1:2), gave methyl 2-[2-(2-propenyl)oxazol-4-yl]-3-[4(methoxycarbonyl)thiazol-2-yl]pyridine-6-carboxylate (21) as a pale yellow oil (21 mg, 79\%). A solution $^{5}$ of osmium(VIII) tetroxide $(1.2 \mathrm{mg}, 4.7 \mu \mathrm{mol})$ in acetonitrile $(60 \mu \mathrm{l})$ was added to a solution of alkene 21 (16 mg, $0.04 \mathrm{mmol})$ in dioxane-water (1:1) (8 ml). Sodium periodate (17 mg, $0.08 \mathrm{mmol})$ was added and the mixture was stirred at room temperature overnight and extracted with dichloromethane $(2 \times 5 \mathrm{ml})$. The combined organic extracts were washed sequentially with saturated aqueous sodium hydrogen carbonate solution $(8 \mathrm{ml})$, water $(8 \mathrm{ml})$ and brine $(8 \mathrm{ml})$, dried $\left(\mathrm{Na}_{2} \mathrm{SO}_{4}\right)$ and evaporated in vасио. Purification by flash chromatography on silica, eluting with light petroleum-ethyl acetate (1:2), gave the title compound as a colourless crystals $(12 \mathrm{mg}, 80 \%)$, mp 159.0-161.0 ${ }^{\circ} \mathrm{C}$ (diethyl ether-hexane) (lit., ${ }^{7} \mathrm{mp} 160.5-161.0{ }^{\circ} \mathrm{C}$ ) (lit., ${ }^{8} \mathrm{mp} 157.3-160 .{ }^{\circ} \mathrm{C}$ ) (Found: $\mathrm{MH}^{+}, 388.0604$. $\mathrm{C}_{17} \mathrm{H}_{13} \mathrm{~N}_{3} \mathrm{O}_{6} \mathrm{~S}$ requires $\left.M H^{+}, 388.0595\right) ; v_{\max }(\mathrm{KBr}) / \mathrm{cm}^{-1} 3150,2954,1728,1702,1573,1534,1477$, $1435,1373,1338,1316,1219,1128,1096,1005,963,869,842,768 ; \delta_{\mathrm{H}}\left(400 \mathrm{MHz} ; \mathrm{CDCl}_{3}\right) 8.33(1 \mathrm{H}$, s, CH), $8.31(1 \mathrm{H}, \mathrm{s}, \mathrm{CH}), 8.16(2 \mathrm{H}$, app s, 4,5-H), 3.98 (3H, s, OMe), 3.91 (3H, s, OMe), $2.41(3 \mathrm{H}, \mathrm{s}$, $\mathrm{Me}) ; \delta_{\mathrm{C}}\left(100 \mathrm{MHz}, \mathrm{CDCl}_{3}\right) 185.6(\mathrm{C}), 164.7$ (C), 164.2 (C), 161.6 (C), 157.0 (C), 148.8 (C), 148.0 (C), $147.2(\mathrm{C}), 142.7(\mathrm{CH}), 140.4(\mathrm{CH}), 140.3(\mathrm{C}), 130.7(\mathrm{C}), 129.7(\mathrm{CH}), 124.2(\mathrm{CH}), 53.3(\mathrm{Me}), 52.8$ (Me), $26.6(\mathrm{Me}) ; m / z(\mathrm{APcI}) 388\left(\mathrm{MH}^{+}, 100 \%\right)$.

\footnotetext{
${ }^{7}$ Abe, H.; Takaishi, T.; Okuda, T. Tetrahedron Lett. 1978, 2791.

${ }^{8}$ Kelly, T. R.; Lang, F. J. Org. Chem. 1996, 61, 4623.
} 


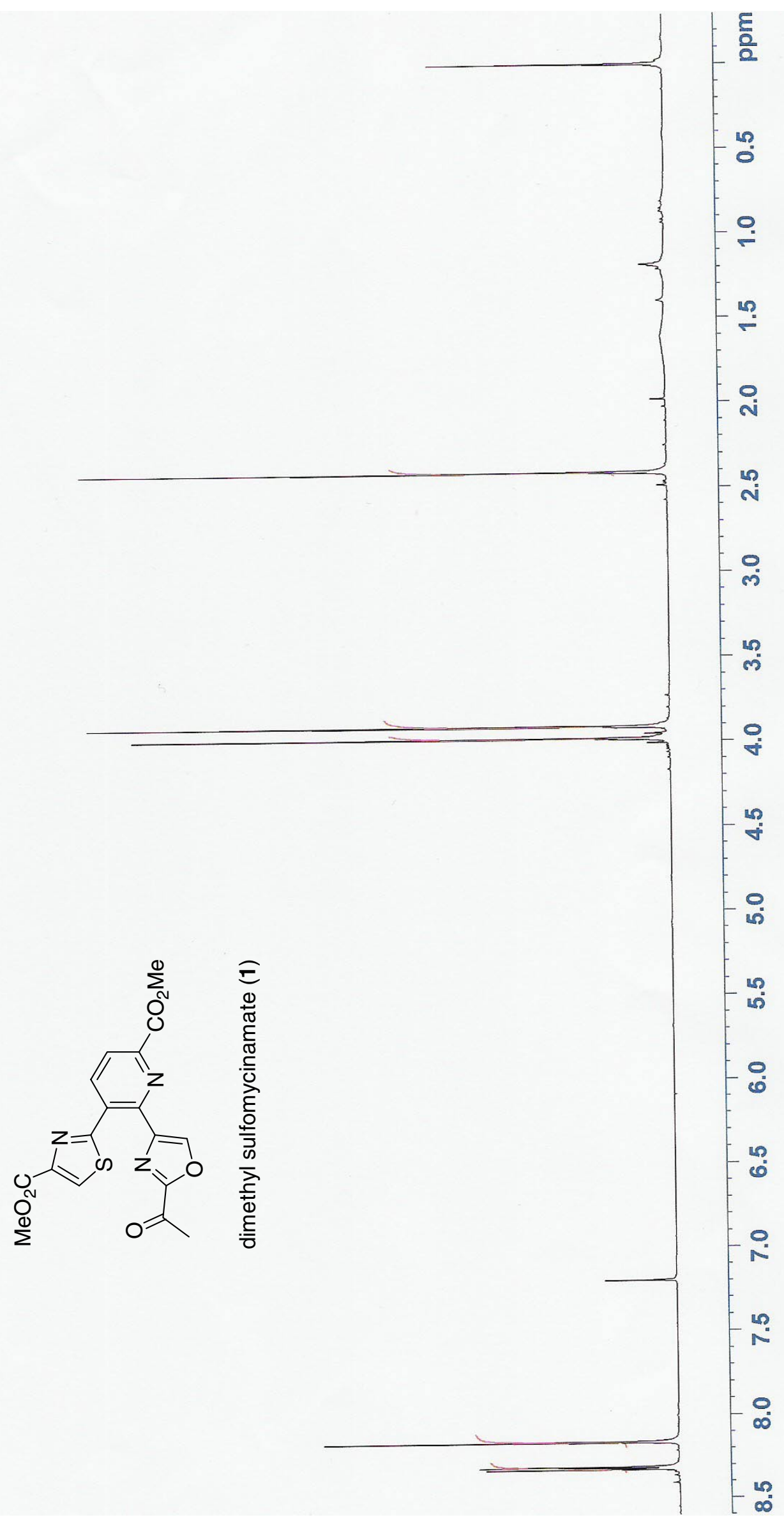




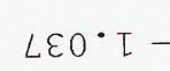

$S \angle G \cdot 9 Z$

29L・29

$\angle 62 \cdot \varepsilon S$

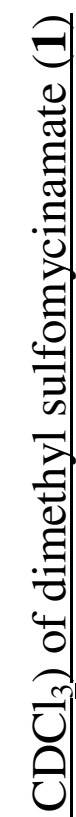

EL・五乙L
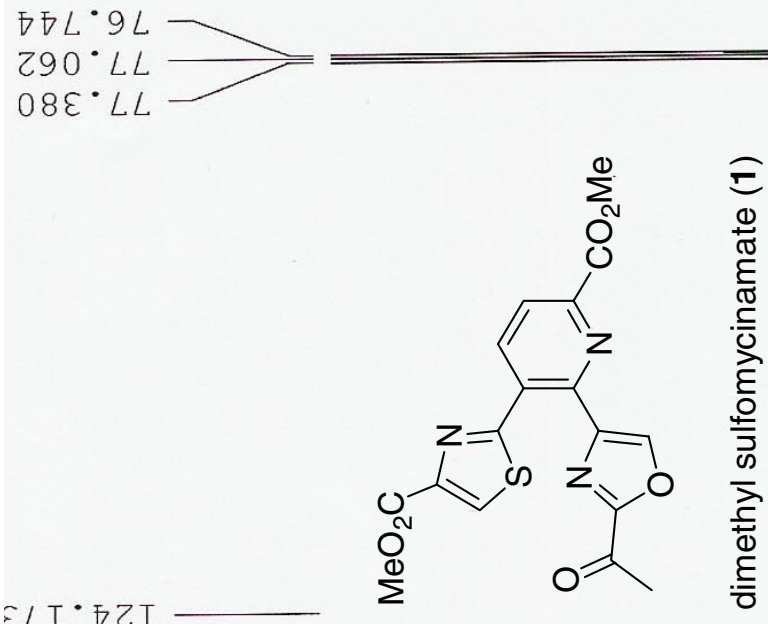

$\varepsilon 0 L \cdot 6 Z I$

$\angle L 9^{\circ} 0 \varepsilon I \longrightarrow$

$\varepsilon 乙 \varepsilon^{\circ} \circ \nabla T$

$\angle L E^{\circ} O D I \longrightarrow$

$099^{\circ} Z \mp I$

$8 \nabla 2^{\circ} \angle D I$

$886^{\circ} \angle T I$

$96 L^{\circ} 8 D I$

I00 $\angle S I$

T99. T9I

$89 I^{\circ}-79 I$

$I Z L \cdot 79 T$

क्षे

¿ $299^{\circ} \mathrm{s} 8 \mathrm{I}$

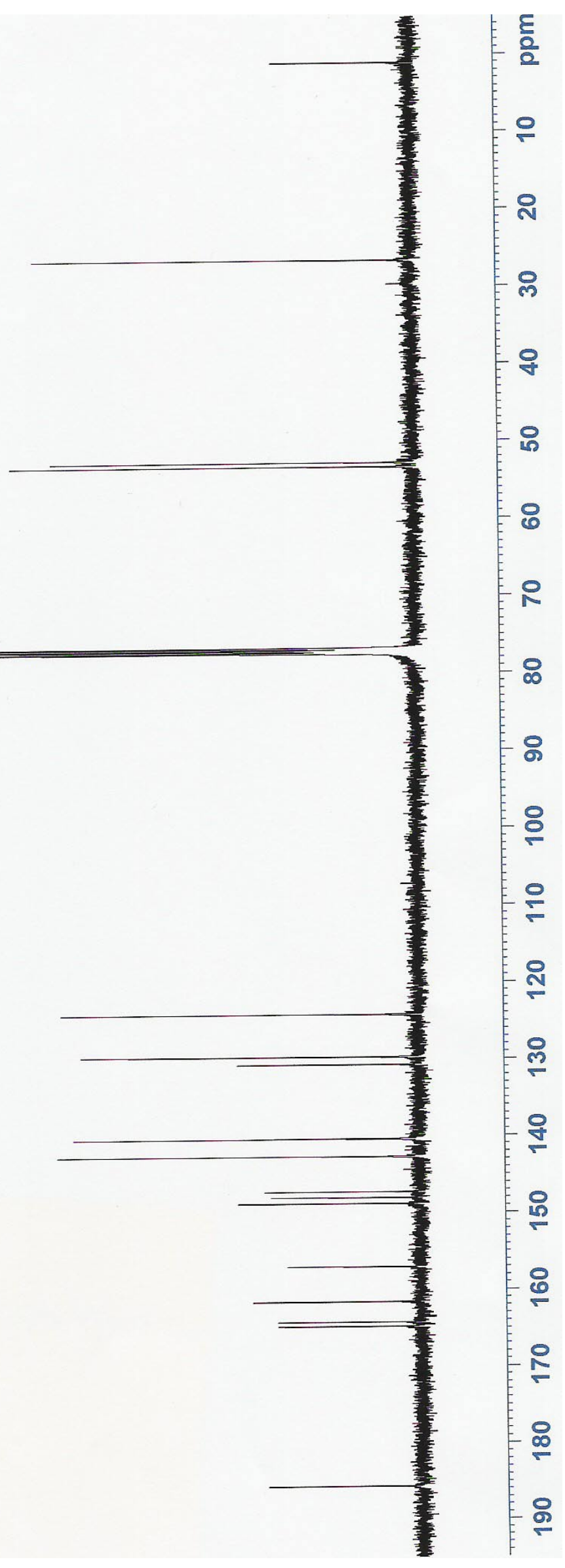

